\title{
stg fimbrial operon from $S$. Typhi STH2370 contributes to association and cell disruption of epithelial and macrophage-like cells
}

\author{
Liliana Berrocal ${ }^{1,2 \dagger}$, Juan A Fuentes ${ }^{1 \dagger}$, A Nicole Trombert ${ }^{1,3}$, Matías R Jofré1,2, Nicolás A Villagra4, \\ Luis M Valenzuela' and Guido C Mora ${ }^{4 *}$
}

\begin{abstract}
Background: Salmonella enterica serovar Typhi (S. Typhi) stg operon, encoding a chaperone/usher fimbria (CU), contributes to an increased adherence to human epithelial cells. However, one report suggests that the presence of the Stg fimbria impairs the monocyte-bacteria association, as deduced by the lower level of invasion to macrophage-like cells observed when the stg fimbrial cluster was overexpressed. Nevertheless, since other CU fimbrial structures increase the entry of S. Typhi into macrophages, and considering that transcriptomic analyses revealed that stg operon is indeed expressed in macrophages, we reassessed the role of the stg operon in the interaction between S. Typhi strain STH2370 and human cells, including macrophage-like cells and mononuclear cells directly taken from human peripheral blood.

Results: We compared S. Typhi STH2370 WT, a Chilean clinical strain, and the S. Typhi STH2370 $\Delta$ stg mutant with respect to association and invasion using epithelial and macrophage-like cells. We observed that deletion of stg operon reduced the association and invasion of S. Typhi, in both cellular types. The presence of the cloned stg operon restored the WT phenotype in all the cases. Moreover, we compared Salmonella enterica sv. Typhimurium 14028s (S. Typhimurium, a serovar lacking stg operon) and S. Typhimurium heterologously expressing S. Typhi stg. We found that the latter presents an increased cell disruption of polarized epithelial cells and an increased association in both epithelial and macrophage-like cells.
\end{abstract}

Conclusions: S. Typhi stg operon encodes a functional adhesin that participates in the interaction bacteria-eukaryotic cells, including epithelial cells and macrophages-like cells. The phenotypes associated to stg operon include increased association and consequent invasion in bacteria-eukaryotic cells, and cell disruption.

Keywords: Salmonella Typhi, stg, Fimbriae, Adherence, Association bacteria-eukaryotic cells

\section{Background}

An essential step in the successful colonization and production of disease by microbial pathogens is their ability to adhere to host cells surfaces [1]. Most pathogens possess many molecules (called adhesins) that mediate

\footnotetext{
*Correspondence: gmora@unab.cl

†Liliana Berrocal and Juan A Fuentes contributed equally to this work ${ }^{4}$ Laboratorio de Microbiología, Facultad de Medicina, Universidad Andres Bello, República 313, Santiago, Chile

Full list of author information is available at the end of the article
}

host adherence. Often these molecules are synergistic in their function, thereby enhancing adherence, while others appear to be functionally redundant [2]. Fimbriae, also called pili, are important adhesins that facilitate host tissue colonization in a large number of pathogens. According to their biosynthetic pathway, fimbriae can be classified into five major groups: curli, type IV pili, type III secretion needle, type IV secretion pili, and chaperone/usher fimbriae (CU fimbriae) [3-6]. Genome analyses of Enterobacteriaceae family members, including 
the host-specific Salmonella enterica serovar Typhi (S. Typhi), revealed that there are at least twelve fimbria operons involved in the CU dependent pathway but only few of them have been characterized to date [7]. Salmonella enterica (S. enterica) serovars, normally acquired by ingesting contaminated water or food, have undergone mutations and horizontal genetic transfer, resulting in unique combinations of fimbrial operons. This presumably contributes to genus evolution and to the rising of different adhesive properties [7, 8]. Actually, sequencing of $S$. Typhi genome reveals twelve operons encoding fimbriae of the CU assembly pathway (i.e. sef, fim, saf, tcf, $b c f, s t a, s t b, s t c, s t d, s t e, s t g$, and $s t h)$, while five of these operons (i.e. sef, $t c f$, sta, ste, and $s t g$ ) are absent from the generalist $S$. enterica serovar Typhimurium $(S$. Typhimurium) [7]. Thus, the differences found between $S$. Typhi and $S$. Typhimurium, including the host-specificity, might be based on the bacteria-host cell interplay. This interaction depends, at least in part, on specific sets of fimbriae contributing to the development of the disease [7].

Among CU fimbrial operons present in $S$. Typhi and absent from $S$. Typhimurium, stg has caught our interest. This operon is constituted by four open reading frames known as $\operatorname{stg} A$ (main fimbrial subunit), $\operatorname{stg} B$ (chaperone), $\operatorname{stg} C$ (outer membrane usher) and $\operatorname{stg} D$ (adhesion tip). Previously, it was reported that stgC (STY3920) contains a premature stop codon that disrupts the predicted open reading frame (ORF) encoding the usher; therefore $s t g C$ was considered a pseudogene [9]. Nevertheless, the stg operon seems to encode a functional fimbria since a $S$. Typhi $\Delta s t g$ mutant exhibits an decreased adherence to human epithelial cells compared with the WT [10]. In contrast, in the same report the authors suggest that the presence of the Stg fimbria impairs the macrophagelike-bacteria association, as deduced by the lower level of invasion to monocytes observed when the $s t g$ fimbrial cluster was overexpressed [10]. Nevertheless, it has been reported that other $\mathrm{CU}$ fimbrial structures increase the entry of $S$. Typhi into macrophages/monocytes [11]. Most importantly, transcriptomic analyses revealed that stg operon is indeed expressed in macrophages, suggesting that this operon might be participating in the interaction with these cells. These data prompted us to reassess the role of the stg operon with respect to the interaction between $S$. Typhi and human cells, including macrophage-like cells and mononuclear cells directly taken from human peripheral blood.

In this work, we determined that the stg operon contributed to increase association of bacteria and both epithelial and macrophage-like cells. Moreover, stg operon contributes to cell invasion and epithelial cell disruption, strongly suggesting that the Stg fimbria are actively participating in different steps of $S$. Typhi infection process.

\section{Results}

The stg operon contributes to the association, invasion, and to an increased permeability of HEp-2 human epithelial cells in S. enterica

Considering that one of the first steps in the S. enterica infection involves the interaction with human epithelial cells, the contribution of the stg operon to cell adherence was assessed using HEp-2 cells. For that, the strains to be tested were cultured in LB to $\mathrm{OD}_{600}=0.2$ in microaerophilia without shaking prior to determining the number of bacteria associated to eukaryotic cells and the number of bacteria that invaded as described in "Methods". Associated bacteria can be defined as adherent bacteria plus bacteria that invaded during the early stage of the interaction between bacteria and eukaryotic cells. As observed in Figure 1a, $S$. Typhi $\triangle \operatorname{stg} A B C D$ (i.e. $\triangle s t g$ ) exhibited

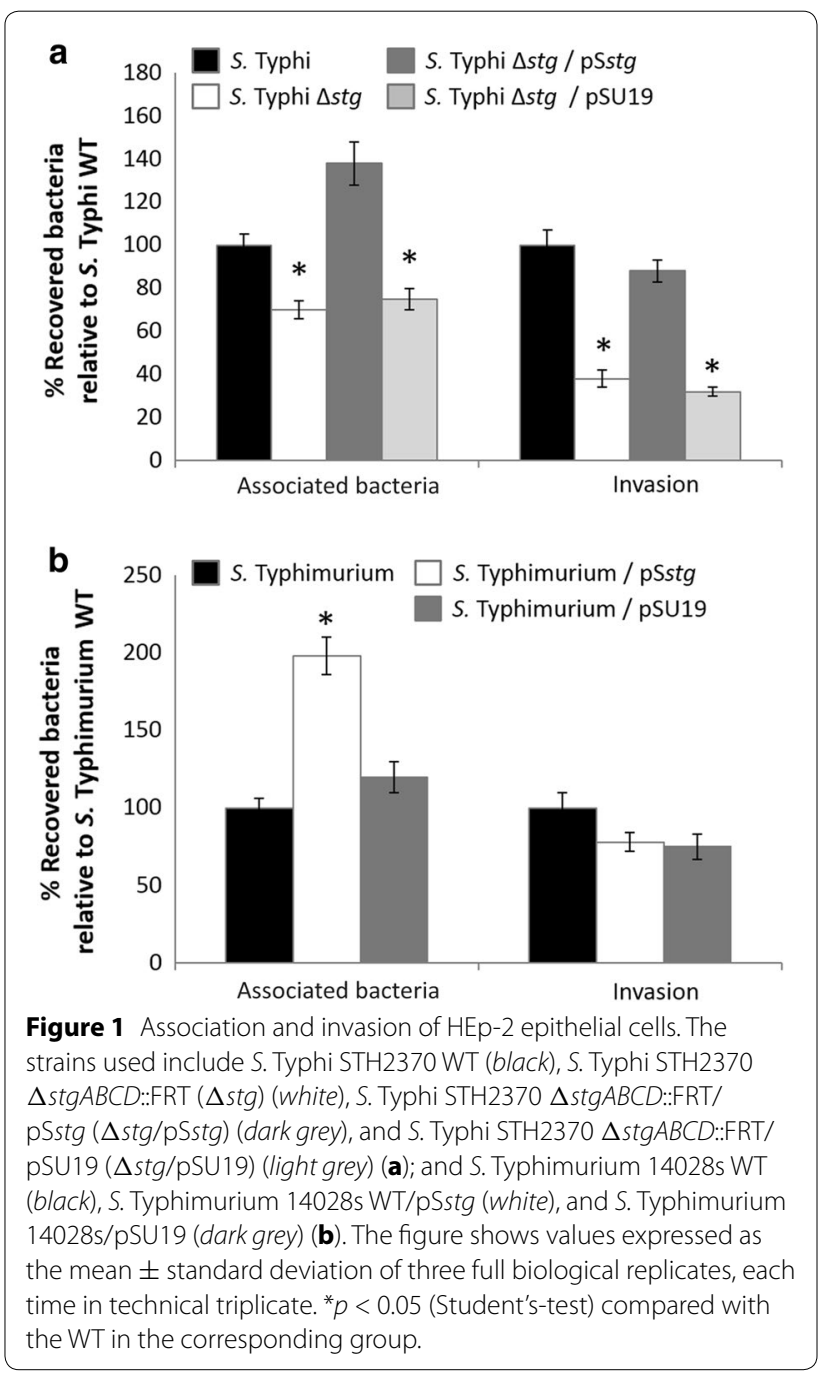


a significantly lower level of association to HEp-2 compared to the otherwise isogenic $S$. Typhi STH2370 WT, a highly virulent Chilean strain [12]. The complementation with the S. Typhi stg whole operon cloned into the pSU19 plasmid (pSstg) restored the WT phenotype, whereas the empty vector pSU19 exerted no effect (Figure 1a; Additional file 1: Table S1). On the other hand, S. Typhimurium naturally lacks the stg operon [7]. Thus, to test the contribution of the stg operon in a heterologous system, we transformed pSstg into S. Typhimurium 14028s WT prior to testing the bacterial association to the HEp-2 cells. As shown in Figure 1b (and Additional file 1: Table S1), S. Typhimurium 14028s WT/pSstg exhibited an increased association compared with the respective $S$. Typhimurium 14028s WT.

Considering that an increased association between bacteria and epithelial cells conceivably may affect the subsequent bacterial internalization, we assessed whether stg was implicated in the invasion of epithelial cells. The invasion is a critical step in the normal infection cycle of $S$. enterica. Since the invasion is promoted by effectors injected into host cells through a type-III secretory system, an increased association bacteria-epithelial cell might affect this process. To test this hypothesis, we performed a gentamicin protection assay comparing the invasion rates of $S$. Typhi STH2370 WT and $S$. Typhi $\Delta s t g$. As shown in Figure 1a, $S$. Typhi $\Delta$ stg presented an impaired invasion compared with the WT strain, consistent with the results obtained for the association bacteria-epithelial cells. Again, the pSstg plasmid, and not the vector alone, restored the WT phenotype (Figure 1a, Additional file 1: Table S1). Nevertheless, when invasion efficiency was calculated by determining the ratio of invaded/associated bacteria using the raw data (Additional file 1: Table S1), no significant differences were observed. This result suggests that the decreased invasion is a consequence of a decreased association, probably mediated by an effect in adherence, and not to an effect in invasion specifically. On the other hand, the pSstg plasmid apparently produced no effects in the invasion of the $S$. Typhimurium heterologous strain (Figure 1b), showing that the stg operon is not specifically contributing to the invasion of $S$. Typhimurium 14028s under the tested conditions.

$S$. Typhi exhibits a high cytotoxicity towards eukaryotic cells compared to $S$. Typhimurium, clearly affecting the permeability of the infected cells. This phenotype can be explained because of the presence of cytolytic factors in the serovar Typhi and/or because of the presence of proteins that diminishes the cell cytotoxicity in the serovar Typhimurium [13-15]. Since the stg operon is only present in the serovar Typhi and absent from the serovar Typhimurium, we assessed whether the presence of the stg operon can also affect the cell permeability of a monolayer of epithelial cells. For that, polarized HT-29 monolayers were cultured in Transwells and incubated for 1 week to allow cell polarization with an apical zone (upper chamber) and a basolateral zone (lower chamber). Cell polarization was confirmed by a gradual increase of transepithelial resistance (TER) (data not shown). The polarized monolayers were subsequently infected with $S$. Typhimurium 14028s WT, $S$. Typhimurium 14028s WT/ pSstg, and S. Typhimurium 14028s WT/pSU19. As control, we used S. Typhi STH2370 WT. The infected monolayers were used to perform a modified transepithelial migration assay that included addition of gentamicin (after $1 \mathrm{~h}$ of infection) into the upper chamber as previously described $[14,15]$. As shown in Figure 2, the recovered $\mathrm{CFU} / \mathrm{mL}$ represented bacteria that migrated to the lower chamber and survived the presence of the gentamicin leaking through the cell monolayer. If bacteria disrupt the integrity of the monolayer, gentamicin will leak through from the upper chamber to the lower chamber, killing bacteria in the lower chamber and decreasing the recovered $\mathrm{CFU} / \mathrm{mL}$. On the other hand, if the monolayer is not disrupted, the recovered $\mathrm{CFU} / \mathrm{mL}$ should remain essentially constant over the same time course since gentamicin cannot permeate through cellular membranes [16]. As observed in Figure 2, the recovered CFU/ $\mathrm{mL}$ corresponding to $S$. Typhimurium 14028s presented a slight decline over the time course of the assay (black squares), showing that the monolayer integrity is not largely affected by bacteria in this case, accordingly to

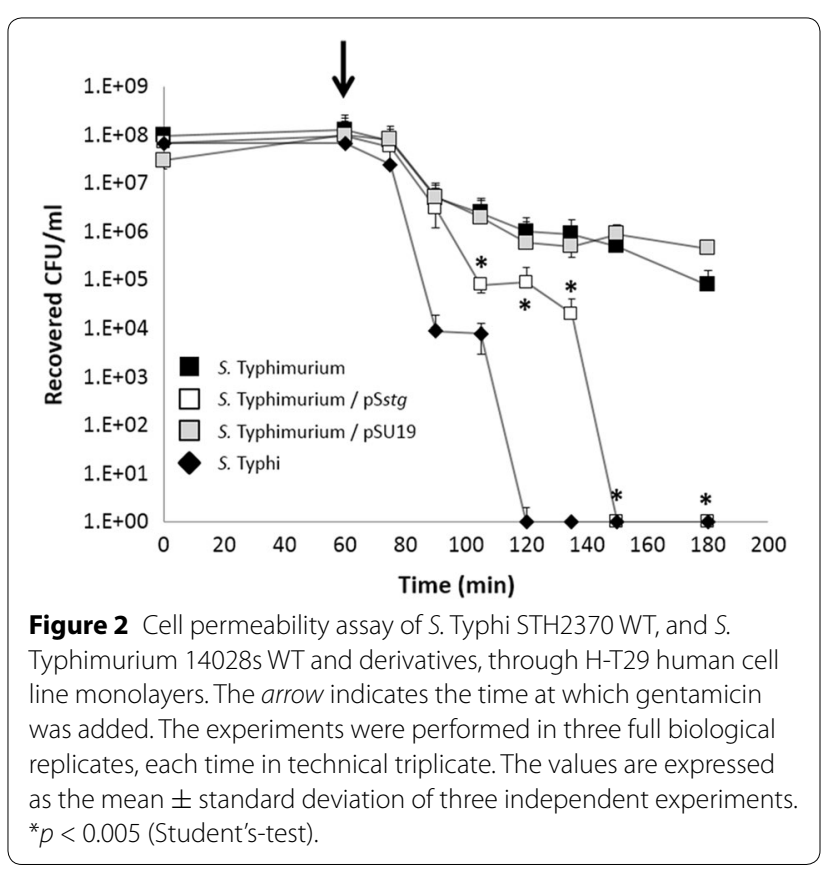


previous published studies $[14,15]$. In contrast, the CFU/ $\mathrm{mL}$ of $S$. Typhi STH2370 recovered from the lower chamber abruptly decreased after the gentamicin addition until they became undetectable, showing that the gentamicin leaked into the lower chamber due to a monolayer disruption (black diamonds). Most importantly, when $S$. Typhimurium 14028s was complemented with the $S$. Typhi stg operon (pSstg, white squares) we observed that the corresponding recovered CFU/mL clearly decreased, marking a sharp difference with the otherwise isogenic $S$. Typhimurium 14028s WT strain and resembling the $S$. Typhi phenotype (compare the black squares and white squares) (Figure 2). All these results were corroborated by measuring the transepithelial electrical resistance (TER) of the infected HT-29 monolayer as previously described [14] (data not shown).

All these results together show that the stg operon contributes to increase the interaction bacteria-epithelial cell and the invasion in $S$. Typhi. Moreover, when heterologously expressed, stg contributes to increase the adherence, and cell disruption in $S$. Typhimurium.

\section{The stg operon contributes to increase the association} of bacteria and human macrophage-like cells in S. enterica Macrophage-like cells play an important role in the dissemination of Salmonella enterica in the systemic phase of the disease [9]. In a previous report, it has been stated that deletion of stg increased uptake of serovar Typhi by human macrophages, and overexpression of stg operon in $S$. Typhi and $S$. Typhimurium strains reduced phagocytosis by human macrophages [10]. In that study, S. Typhi strain ISP1820 and derivatives were grown to stationary phase under aerobic conditions, prior to performing the infection [10]. Nevertheless, other studies reported that the stg operon increases its expression during an infection of macrophages $[17,18]$, suggesting that this operon may contribute to $S$. Typhi invasion in these cells. Considering that other $\mathrm{CU}$ fimbriae have an active role in bacterial invasion of host phagocytic cells [11, 19], we reassessed the role of the stg operon in the macrophage-like cell interaction. The first approach was to determine the role of $s t g$ in the bacterial association to human macrophagelike cells (monocytes). For that, the human monocyte cell line U937 was infected with $S$. Typhi STH2370 WT, S. Typhi STH2370 $\Delta s t g, S$. Typhi STH2370 $\Delta s t g / p S s t g$, or $S$. Typhi STH2370 $\Delta s t g /$ pSU19, previously cultured in $\mathrm{LB}$ to $\mathrm{OD}_{600}=0.2$ under microaerophilic conditions, to perform the adherence and invasion assays. As shown in Figure 3a and in Additional file 1: Table S2, S. Typhi $\Delta$ stg exhibited a significant impaired association to U937 cells compared to the WT strain. Trans-complementing with the pSstg plasmid reverted the mutant phenotype (Figure $3 \mathrm{a})$. Considering that a lower association could lead

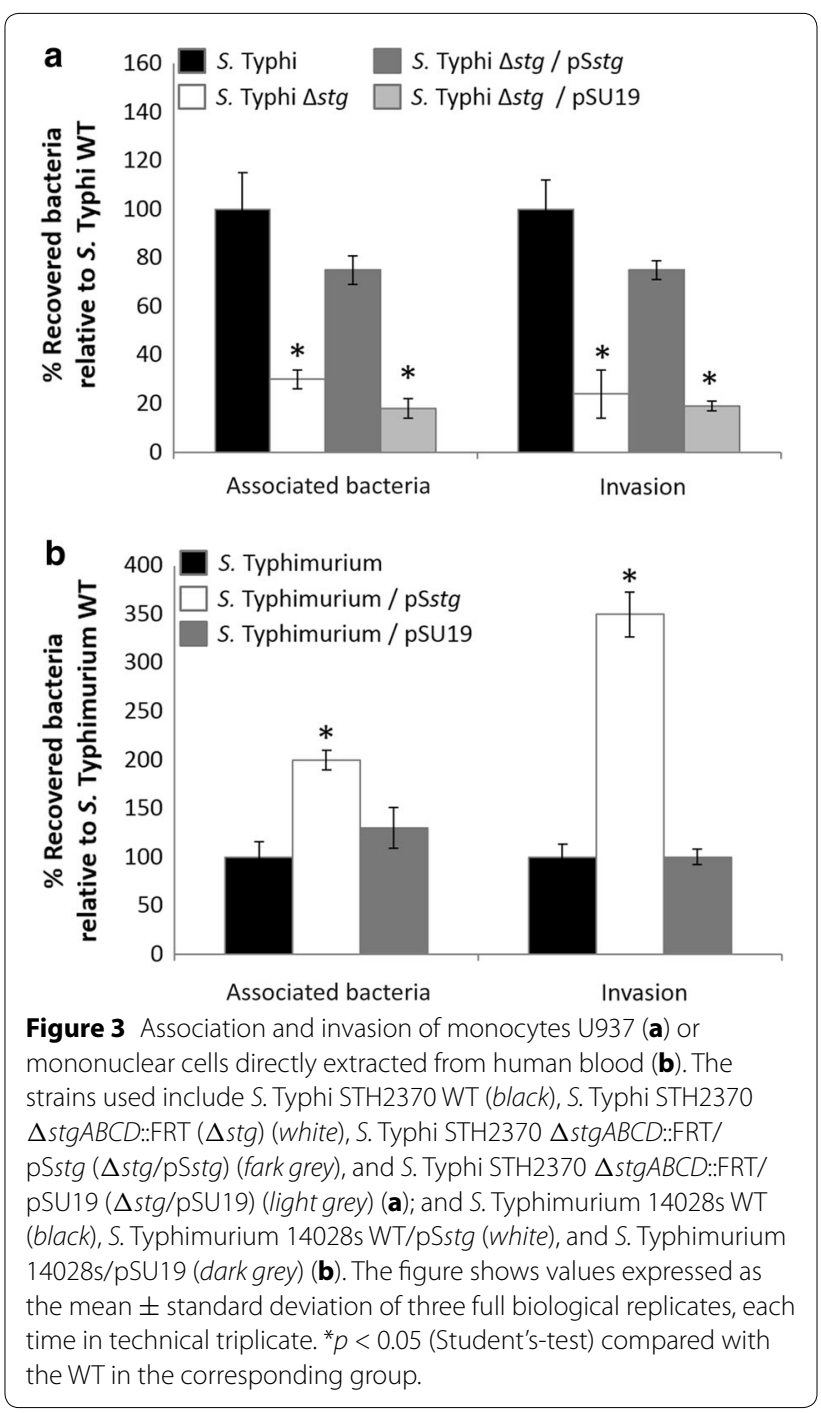

to a decreased bacterial invasion, we determined the bacterial uptake by U937 monocytes using the gentamicin protection assay. As observed in Figure 3a, the $S$. Typhi $\Delta s t g$ also presented a decreased invasion compared with the WT. Again, when invasion efficiency was calculated (see data in Additional file 1: Table S2), no significant differences were observed, suggesting that the contribution of $s t g$ to the invasion depends on the association (plausibly on the adherence) in this case.

To assess the contribution of the stg operon in macrophage-like-bacteria interaction in an independent way, we performed association and invasion assays using $S$. Typhimurium heterologously expressing S. Typhi stg from the pSstg plasmid. In this case, we tested the $S$. Typhimurium/pSstg with a mononuclear cell fraction obtained directly from human peripheral blood isolated with HISTOPAQUE'-1077. As observed in Figure $3 \mathrm{~b}$ and in Additional file 1: Table S3, the presence of the stg 
operon in $S$. Typhimurium significantly contributes to the bacterial uptake compared with the control. In all cases, the empty vector exerted no effects (Figure 3 ). The invasion efficiency (Additional file 1: Table S3) presented no significant differences among the strains, suggesting that also in this case the contribution of stg to invasion is dependent on the bacteria-eukaryotic cells association.

All these results together show that the stg operon contributes to the macrophage-like-bacteria interaction, contributing to the association and the invasion in a line of human monocytes (i.e. U937) and in mononuclear cells directly extracted from human blood.

\section{Discussion}

The adherence is a critical process for host colonization and disease production in several pathogens, including S. enterica [1]. In this work, we found that stg operon contributes to adherence/invasion of $S$. Typhi; and to adherence/invasion, and cell disruption when heterologously expressed in $S$. Typhimurium. These findings strongly suggest that $\operatorname{stg} A B C D$ encode a functional fimbria that is able to assemble under the growth conditions used. Nevertheless, the $\operatorname{stg} C$ gene, encoding the putative usher for the fimbria assembly, contains an internal stop codon reported for $S$. Typhi CT18; therefore it has been considered as a pseudogene (i.e. non-functional) [7]. We sequenced an internal fragment of the $\operatorname{stg} C$ gene of the Chilean clinical strain S. Typhi STH2370 [12], a highly virulent isolate, and we observed that the stop codon is at the same position observed in the sequenced strain S. Typhi CT18 (data not shown). A non-functional $s t g C$ could preclude the fimbrial assembly, affecting the phenotypes observed in this work. However, despite the presence of the single internal stop codon in $\operatorname{stg} C$, a fully functional StgC protein might be produced. In some cases, single premature stop codons can be hopped by ribosomes, allowing a normal protein synthesis in $S$. enterica [20], similarly to some phage genes containing TGA premature stop codons [21]. This same mechanism might occur in $\operatorname{stg} C$ gene to produce a functional usher protein. Another explanation could be the complementation of the StgC function (i.e. usher) by functional ushers from other chaperon-usher operons found in S. enterica, due to the high identity found among them [7]. Nevertheless, more experiments have to be performed in order to test these hypotheses.

Here, we also showed that the stg operon, present in $S$. Typhi and absent from $S$. Typhimurium, contributes to the interaction between $S$. enterica and host cells, including epithelial cells and macrophage-like cells. In the epithelial cell line HEp-2, we observed that stg contributed to the bacterial association to epithelial cells, suggesting that stg could be important in the first steps of S. enterica infection, accordingly to previously reported studies that showed an impaired adherence of $S$. Typhi $\Delta s t g$ mutant to the INT-407 epithelial cell line [10]. We also found that the increased association due to the presence of the stg operon is accompanied with an improved invasion of HEp-2 cells. Nevertheless, Forest et al. showed that apparently the stg operon is not contributing to this process [10], similarly to the observed with $S$. Typhimurium heterologously expressing stg (Figure 1b). This difference could be explained by the fact that we cultured bacteria under microaerophilic conditions prior to performing the invasion assay, as previously described [16]. In contrast, Forest et al. [10] cultured bacteria to stationary phase with aeration. The microaerophilic condition is an important inducer of the invasion process in S. enterica (unpublished results and [22]). Thus, the bacterial culture condition seems to be a key factor to assess the interaction between bacteria and eukaryotic cells. On the other hand, the null contribution of stg to the $S$. Typhimurium invasion could explain why this serovar lacks the stgABCD operon [7].

Cell permeability in HT-29 epithelial cells was also affected by the presence of the stg operon in $S$. Typhimurium (Figure 2), plausibly due to cell disruption [14]. Some authors have suggested that $S$. Typhi has been selected for an increased disruptive ability towards epithelial cells compared to $S$. Typhimurium in order to perform a successful systemic/deeper infection in humans $[14,15]$. Nevertheless, we found no differences between $S$. Typhi WT and $S$. Typhi $\Delta s t g$ with respect to the cells disruption (data not shown). This result may be explained by the fact that $S$. Typhi presents several mechanisms to disrupt cells. Actually, S. Typhi presents other factors contributing to increase the ability to disrupt epithelial cells. Some of these factors, as well as stg, are absent from $S$. Typhimurium, including $h l y E$, and sseJ and sopD2 pseudogenes [13-15]. However, how stg is improving cell disruption in $S$. Typhimurium is unknown. We speculate that stg contributes to this phenotype by increasing the bacteria-cell contact. Thus, the results showed in this paper indicate that the stg fimbrial operon has a role in association, invasion, and in disruption of epithelial cells in S. enterica.

During the $S$. Typhi infectious process, bacteria interact with phagocytic cells that play a crucial role in bacterial systemic dissemination and possibly in host specificity $[10,23]$. Our results with monocyte U937 cells and with human mononuclear cells suggested a role of the stg operon in bacterial association and invasion of phagocytic cells. These results are consistent with works showing the expression of stg fimbrial genes in human macrophages $[17,18]$. Other chaperon-usher fimbriae, such as sef operon, proved to be involved in bacterial interaction with 
phagocytic cells during peritoneal infection in Salmonella enterica, reinforcing the fact that this kind of fimbriae could contribute to the systemic spreading [11]. In contrast, Forest et al. [10] reported that loss of stg results in increased phagocytosis of serovar Typhi by THP-1 human macrophages-like cells. Again, this apparent contradiction to our findings could be explained by the bacterial growth conditions used before infecting monocytes. Forest et al. used bacteria grown overnight at $37^{\circ} \mathrm{C}$ before infection of cells [10], while we used bacteria grown to $\mathrm{OD}_{600}=0.2$ under microaerophilic conditions without shaking, a condition promoting an efficient invasion to macrophages $[24,25]$. Finally, it is important to remark that Forest et al. [10] worked with $S$. Typhi strain ISP1820, whereas, in this manuscript, we report a study performed with $S$. Typhi strain STH2370 [12], a Chilean clinical isolate. Considering that $S$. Typhi STH2370 presents some genomic differences compared with other standard $S$. Typhi strain such as CT18 and Ty2 (unpublished data and [12, 26, 27]), it is also possible that the differences between our results and previous data are due to the bacterial strains tested.

As stated above, stg is contributing to bacterial interaction with both epithelial cells and macrophages-like cells, strongly suggesting that the stg operon could participate in the intestinal step of infection as well as in the systemic spread. It has been described other virulence factors not circumscribed to a unique stage of the infection. For example, Salmonella SipA, encoded by SPI-1 enhances entry to intestinal epithelial cells by promoting actin polymerization, but also contributing to proliferation of S. enterica within macrophages [28]. Moreover, other genes belonging to SPI-1 such as prg, sip, spa, and $i n v$ genes increase their expression inside the gut and also during early stages of bacterial uptake by human macrophages [18]. The possible role of stg operon in both stages of $S$. Typhi infection (i.e. intestinal and systemic) suggests that it may encode an important factor for the pathogenesis of this human-specific pathogen.

\section{Conclusions}

$S$. Typhi stg operon, encoding a functional adhesin, contributes to the interaction of bacteria and eukaryotic cells, including epithelial and macrophages-like cells. The phenotypes associated to stg operon include stg operon include increased association and consequent invasion in bacteria-eukaryotic cells, and cell disruption.

\section{Methods}

\section{Culture conditions}

Clinical isolate of $S$. Typhi strain STH2370, a highly virulent strain from the Infectious Diseases Hospital Lucio Córdova in Santiago, Chile. The $S$. Typhimurium strains used in this study are derivatives of the parental wild-type strain ATCC14028s. The strains were routinely grown in Luria-Bertani (LB) broth (Bacto tryptone, $10 \mathrm{~g} / \mathrm{L}$; Bacto yeast extract, $5 \mathrm{~g} / \mathrm{L}$; and $\mathrm{NaCl}, 5 \mathrm{~g} / \mathrm{L}$ ) at $37^{\circ} \mathrm{C}$ with shaking. For assessing bacteria-eukaryotic cells association and for the invasion assays, bacteria were cultured in LB broth at $37^{\circ} \mathrm{C}$ in microaerophilia as previously described [16]. Culture media were supplemented with chloramphenicol $(20 \mu \mathrm{g} / \mathrm{mL})$, ampicillin $(100 \mu \mathrm{g} / \mathrm{mL})$ or kanamycin $(50 \mu \mathrm{g} / \mathrm{mL})$ when required. Solid media contain Bacto agar (Merck) at $15 \mathrm{~g} / \mathrm{L}$.

\section{Construction of mutants}

S. Typhi $\triangle \operatorname{stg} A B C D:$ :FRT (or simply named $S$. Typhi $\Delta s t g$ ) was constructed using the Red-Swap procedure as described [29]. The primers used in the lambda red recombination system correspond to WNstgA (AATGA AACATCGGGATATATAATATATCAATAGAGTT ATACATATGAATATCCTCCTTAG) and WCstgD (TTGA TATGAC T TAT T T TG TAAT TAGAT TAGT TCG T TATTTTGTAGGCTGGAGCTGCTTCG).

\section{PCR amplifications and plasmid construction}

PCR amplifications were performed using an Eppendorf thermal cycler and Accuprime Taq DNA polymerase (Invitrogen). Reaction mixtures contained 1X PCR buffer, $1.5 \mathrm{mM} \mathrm{MgCl}$, each dNTP $(200 \mu \mathrm{M})$, primers $(1 \mu \mathrm{M}), 100 \mathrm{ng}$ of template DNA, and $2 \mathrm{U}$ polymerase. Standard conditions for amplification were 30 cycles at $96^{\circ} \mathrm{C}$ for $40 \mathrm{~s}, 55^{\circ} \mathrm{C}$ for $45 \mathrm{~s}$, and $72^{\circ} \mathrm{C}$ for $2 \mathrm{~min}$, followed by a final extension step at $72^{\circ} \mathrm{C}$ for $10 \mathrm{~min}$. Template $S$. Typhi STH2370 (WT) chromosomal DNA was prepared as described [30]. The pSstg plasmid was constructed by digesting the pGstg with EcoRI and cloning the fragment that contained the stg operon into the EcoRI site in midcopy vector pSU19. Primers used for stg operon amplification were NstgA (AGCGACAACATGCACATCAT), and CstgD (CAGGGCCGTAGTTCTTGAT). Products generated by PCR amplification were resolved in $0.8 \%$ agarose gels.

\section{Determination of bacteria: eukaryotic cell association and invasion assays}

Macrophage-like human cell line (U937), and the epithelial cell lines (HEp-2 and HT-29) were maintained in RPMI 1640 (Invitrogen) containing 10\% fetal calf serum pre-treated for $30 \mathrm{~min}$ at $60^{\circ} \mathrm{C}$, at $37^{\circ} \mathrm{C}$ in a $5 \%$ $\mathrm{CO}_{2} / 95 \%$ air mixture. Peripheral mononuclear cells were obtained from healthy people using HISTOPAQUE -1077 according the protocol described by Boyum et al. [31]. For assessing the bacteria-epithelial cell association, HEp-2 cells were seeded at a concentration of $5 \times 10^{5}$ cells per well in 96-well microtiter plates. Bacteria were grown in microaerophilia as 
previously described [16] in static conditions to an $\mathrm{OD}_{600}=0.2$ prior to being added to a cell monolayer at a MOI of 100:1. After incubation for $1 \mathrm{~h}$ at $37^{\circ} \mathrm{C}$, the infected cells were washed five times with sterile PBS. The eukaryotic cells were disrupted with sodium deoxycholate $0.5 \%$ prior to determining the number of bacteria associated to the eukaryotic cells by bacterial plate counting (CFU). The level of associated bacteria was expressed as a percentage of the initial inoculum. For monocytes cell association assays, U937 cells were seeded at a concentration of $5 \times 10^{5}$ cells in $1.5 \mathrm{~mL}$ tubes. Bacteria were grown in microaerophilia as previously described [16] in static conditions to an $\mathrm{OD}_{600}=0.2$ prior to being added to macrophages at a MOI of 100:1. The mixture U937 and bacteria was centrifuged for $5 \mathrm{~min}$ at $1,000 \times g$ to synchronize the bacteria-cell interaction. After incubation for $1 \mathrm{~h}$ at $37^{\circ} \mathrm{C}$, the infected cells were washed five times with sterile PBS prior to adding 0.5\% deoxycholic acid sodium salt in PBS. Bacteria associated to eukaryotic cells were determined by bacterial plate counting (CFU). The level of association was expressed as a percentage of the initial inoculum. To determine the invasion rate to HEp-2 epithelial cells, the U937 macrophage-like cells, or mononuclear cells, the gentamicin protection assay was performed as previously described [13].

\section{Transepithelial migration assay}

Colon carcinoma HT29 cells were grown to confluence on $3.0 \mathrm{~mm}$ pore-size filters ("Transwells", Millicell', Millipore) in glucose-free RPMI medium (Gibco) for 2 weeks at $37^{\circ} \mathrm{C}$ and $5 \%$ of $\mathrm{CO}_{2}$ to allow cell polarization, which was subsequently confirmed by a gradual increase of transepithelial resistance (TER). Transwells with cellular monolayers were inoculated apically with $400 \mu \mathrm{L}$ of bacterial cultures at approximately $1 \times 10^{7} \mathrm{CFU} / \mathrm{mL}$ previously grown in microaerophilia [16] to and $\mathrm{OD}_{600}=0.2 \mathrm{in}$ LB. After $1 \mathrm{~h}$ incubation at $37^{\circ} \mathrm{C}$ to allow bacterial uptake by the human cells, monolayers were washed once with sterile PBS and $400 \mu \mathrm{L}$ of glucose-free RPMI medium containing gentamicin $(50 \mu \mathrm{g} / \mathrm{mL})$ was added to eliminate extracellular bacteria. Aliquots $(20 \mu \mathrm{L})$ from the basal compartment (lower chamber) were then collected every 15 min and up to $3 \mathrm{~h}$ of incubation to monitor the number of bacteria that crossed the cell monolayer as determined by CFUs count on LB agar plates. All the results obtained with the transepithelial migration assay were corroborated by measuring the transepithelial electrical resistance as previously described [14].

\section{Consent}

All the human subjects that donated blood presented an informed consent to participate in this study.

\section{Statistics}

All results are expressed as the mean \pm SD of an individual experiment performed in triplicate. $p$ values were calculated according to Student's t test, and a value of $p<0.05$ was considered to be statistically significant.

\section{Additional file}

Additional file 1: Association or invasion of different eukaryotic cells, including HEp-2 (epithelial cells), U937 (macrophage-like cells), and mononuclear cells directly extracted from human blood. The bacterial strains used include S. Typhi STH2370 WT, S. Typhi STH2370 $\Delta$ stgABCD::FRT ( $\Delta$ stg), S. Typhi STH2370 $\Delta$ stgABCD::FRT/pSstg ( $\Delta$ stg/pSstg), and S. Typhi STH2370 $\triangle$ stgABCD::FRT/pSU19 ( $\Delta$ stg/pSU19); and S. Typhimurium 14028s WT, S. Typhimurium 14028s WT/pSstg, and S. Typhimurium 14028s/pSU19. The tables show values expressed as the means \pm standard deviation of 3 full biological replicates, each time in technical triplicate. ${ }^{*} p<0.05$ (Student'sTest) compared with the WT in the corresponding group.

\section{Abbreviations}

S. enterica: Salmonella enterica; S. Typhi: Salmonella enterica serovar Typhi; S. Typhimurium: Salmonella enterica serovar Typhimurium; stg: stgABCD operon.

\section{Authors' contributions}

LB designed the studies and performed the experiments. JAF wrote the paper and made all the figures. ANT performed the transepithelial electrical resistance experiment. MRJ, NV, and LMV contributed to the discussion of results and to the revision of the manuscript. GCM participated in the revision of the manuscript. All authors read and approved the final manuscript.

\section{Author details}

'Laboratorio de Microbiología, Facultad de Ciencias Biológicas, Universidad Andres Bello, República 217, Santiago, Chile. ${ }^{2}$ Present Address: Escuela de Medicina, Facultad de Medicina, Universidad Finis Terrae, Santiago, Av. Pedro de Valdivia 1509, Providencia, Santiago, Chile. ${ }^{3}$ Present Address: Centro de Genómica y Bioinformática, Facultad de Ciencias, Universidad Mayor, Santiago, Camino La Pirámide 5750, Huechuraba, Santiago, Chile. ${ }^{4}$ Laboratorio de Microbiología, Facultad de Medicina, Universidad Andres Bello, República 313, Santiago, Chile.

\section{Acknowledgements}

This work was supported by National Fund for Development of Science and Technology (FONDECYT), Government of Chile, grants 1151393 (G.C.M), 11121506 (J.A.F.), and Núcleo UNAB 22-12/N (G.C.M. and J.A.F.). L.B. was supported by UNAB Grant DI-11-06/I.

\section{Compliance with ethical guidelines}

\section{Competing interests}

The authors declare that they have no competing interests.

Received: 24 March 2015 Accepted: 23 June 2015

Published online: 07 July 2015

\section{References}

1. Godaly G, Frendeus B, Proudfoot A, Svensson M, Klemm P, Svanborg C. Role of fimbriae-mediated adherence for neutrophil migration across Escherichia coli-infected epithelial cell layers. Mol Microbiol. 1998;30(4):725-35.

2. Wagner $\mathrm{C}$, Hensel M. Adhesive mechanisms of Salmonella enterica. Adv Exp Med Biol. 2011;715:17-34. doi:10.1007/978-94-007-0940-9_2.

3. Thanassi DG, Saulino ET, Hultgren SJ. The chaperone/usher pathway: a major terminal branch of the general secretory pathway. Curr Opin Microbiol. 1998;1(2):223-31. 
4. Wu H, Fives-Taylor PM. Molecular strategies for fimbrial expression and assembly. Crit Rev Oral Biol Med Off Publ Am Assoc Oral Biol. 2001;12(2):101-15.

5. van der Woude MW, Baumler AJ. Phase and antigenic variation in bacteria. Clin Microbiol Rev. 2004;17(3):581-611. doi:10.1128/CMR.17.3.581611.2004 table of contents.

6. Waksman G, Hultgren SJ. Structural biology of the chaperone-usher pathway of pilus biogenesis. Nat Rev Microbiol. 2009;7(11):765-74. doi:10.1038/nrmicro2220.

7. Townsend SM, Kramer NE, Edwards R, Baker S, Hamlin N, Simmonds $M$, et al. Salmonella enterica serovar Typhi possesses a unique repertoire of fimbrial gene sequences. Infect Immun. 2001;69(5):2894-901. doi:10.1128/IAl.69.5.2894-2901.2001.

8. Baumler AJ. The record of horizontal gene transfer in Salmonella. Trends Microbiol. 1997;5(8):318-22. doi:10.1016/S0966-842X(97)01082-2.

9. Edwards RA, Matlock BC, Heffernan BJ, Maloy SR. Genomic analysis and growth-phase-dependent regulation of the SEF14 fimbriae of Salmonella enterica serovar Enteritidis. Microbiology. 2001;147(Pt 10):2705-15.

10. Forest C, Faucher SP, Poirier K, Houle S, Dozois CM, Daigle F. Contribution of the stg fimbrial operon of Salmonella enterica serovar Typhi during interaction with human cells. Infect Immun. 2007;75(11):5264-71. doi:10.1128/IAl.00674-07.

11. Edwards RA, Schifferli DM, Maloy SR. A role for Salmonella fimbriae in intraperitoneal infections. Proc Natl Acad Sci USA. 2000;97(3):1258-62.

12. Valenzuela C, Ugalde JA, Mora GC, Álvarez S, Contreras I, Santiviago CA. Draft genome sequence of Salmonella enterica Serovar Typhi Strain STH2370. Genome Announc. 2014;2(1). doi:10.1128/genomeA.00104-14.

13. Fuentes JA, Villagra N, Castillo-Ruiz M, Mora GC. The Salmonella Typhi hlyE gene plays a role in invasion of cultured epithelial cells and its functional transfer to $S$. Typhimurium promotes deep organ infection in mice. Res Microbiol. 2008;159(4):279-87. doi:10.1016/j.resmic.2008.02.006.

14. Trombert AN, Berrocal L, Fuentes JA, Mora GC. S. Typhimurium sseJ gene decreases the S. Typhi cytotoxicity toward cultured epithelial cells. BMC Microbiol. 2010;10:312. doi:10.1186/1471-2180-10-312.

15. Trombert AN, Rodas PI, Mora GC. Reduced invasion to human epithelial cell lines of Salmonella enterica serovar Typhi carrying S. Typhimurium sopD2. FEMS Microbiol Lett. 2011;322(2):150-6. doi:10.1111/j.1574-6968.2011.02347.x.

16. Contreras I, Toro CS, Troncoso G, Mora GC. Salmonella typhi mutants defective in anaerobic respiration are impaired in their ability to replicate within epithelial cells. Microbiology. 1997;143(Pt 8):2665-72.

17. Faucher SP, Curtiss R 3rd, Daigle F. Selective capture of Salmonella enterica serovar typhi genes expressed in macrophages that are absent from the Salmonella enterica serovar Typhimurium genome. Infect Immun. 2005;73(8):5217-21. doi:10.1128/IAI.73.8.5217-5221.2005.

18. Faucher SP, Porwollik S, Dozois CM, McClelland M, Daigle F. Transcriptome of Salmonella enterica serovar Typhi within macrophages revealed through the selective capture of transcribed sequences. Proc Natl Acad Sci USA. 2006;103(6):1906-11. doi:10.1073/pnas.0509183103.
19. Pan Q, Zhang XL, Wu HY, He PW, Wang F, Zhang MS, et al. Aptamers that preferentially bind type IVB pili and inhibit human monocytic-cell invasion by Salmonella enterica serovar Typhi. Antimicrob Agents Chemother. 2005:49(10):4052-60. doi:10.1128/AAC.49.10.4052-4060.2005.

20. Rodas PI, Trombert AN, Mora GC. A holin remnant protein encoded by STY1365 is involved in envelope stability of Salmonella enterica serovar Typhi. FEMS Microbiol Lett. 2011;321(1):58-66. doi:10.1111/j.1574-6968.2011.02310.x.

21. Goldman E, Korus M, Mandecki W. Efficiencies of translation in three reading frames of unusual non-ORF sequences isolated from phage display. FASEB J Off Publ Fed Am Soc Exp Biol. 2000;14(3):603-11.

22. Khullar M, Singh RD, Smriti M, Ganguly NK. Anaerobiosis-induced virulence of Salmonella enterica subsp. enterica serovar Typhimurium: role of phospholipase Cgamma signalling cascade. J Med Microbiol. 2003;52(Pt 9):741-5.

23. Jones BD, Ghori N, Falkow S. Salmonella Typhimurium initiates murine infection by penetrating and destroying the specialized epithelial M cells of the Peyer's patches. J Exp Med. 1994;180(1):15-23.

24. Singh RD, Khullar M, Ganguly NK. Role of anaerobiosis in virulence of Salmonella Typhimurium. Mol Cell Biochem. 2000;215(1-2):39-46.

25. Fink RC, Evans MR, Porwollik S, Vazquez-Torres A, Jones-Carson J, Troxell $B$, et al. FNR is a global regulator of virulence and anaerobic metabolism in Salmonella enterica serovar Typhimurium (ATCC 14028s). J Bacteriol. 2007;189(6):2262-73. doi:10.1128/JB.00726-06.

26. Parkhill J, Dougan G, James KD, Thomson NR, Pickard D, Wain J, et al. Complete genome sequence of a multiple drug resistant Salmonella enterica serovar Typhi CT18. Nature. 2001;413(6858):848-52. doi:10.1038/35101607.

27. Deng W, Liou SR, Plunkett G 3rd, Mayhew GF, Rose DJ, Burland V, et al. Comparative genomics of Salmonella enterica serovar Typhi strains Ty2 and CT18. J Bacteriol. 2003;185(7):2330-7.

28. Brawn LC, Hayward RD, Koronakis V. Salmonella SPI1 effector SipA persists after entry and cooperates with a SPI2 effector to regulate phagosome maturation and intracellular replication. Cell Host Microbe. 2007;1(1):6375. doi:10.1016/j.chom.2007.02.001.

29. Datsenko KA, Wanner BL. One-step inactivation of chromosomal genes in Escherichia coli K-12 using PCR products. Proc Natl Acad Sci USA. 2000;97(12):6640-5. doi:10.1073/pnas.120163297.

30. Santiviago CA, Toro CS, Hidalgo AA, Youderian P, Mora GC. Global regulation of the Salmonella enterica serovar Typhimurium Major Porin, OmpD. J Bacteriol. 2003;185(19):5901-5. doi:10.1128/jb.185.19.5901-5905.2003.

31. Boyum A. Isolation of leucocytes from human blood. A two-phase system for removal of red cells with methylcellulose as erythrocyte-aggregating agent. Scand J Clin Lab Investig Suppl. 1968;97:9-29.

\section{Submit your next manuscript to BioMed Central and take full advantage of:}

- Convenient online submission

- Thorough peer review

- No space constraints or color figure charges

- Immediate publication on acceptance

- Inclusion in PubMed, CAS, Scopus and Google Scholar

- Research which is freely available for redistribution

Submit your manuscript at

www.biomedcentral.com/submit

C Biomed Central 